\title{
Identification of medicinal plant Schisandra chinensis using a potential DNA barcode ITS2
}

\author{
Xian-kuan Li, Bing Wang*, Rong-chun Han, Yan-chao Zheng, Hai-bo Yin Yin, Liang Xu, Jian-kui Zhang, Bao-li Xu \\ Pharmacy College, Liaoning University of Traditional Chinese Medicine, China
}

\begin{abstract}
To test whether the internal transcribed spacer 2 (ITS2) region is an effective marker for using in authenticating of the Schisandra chinensis at the species and population levels, separately. And the results showed that the wild populations had higher percentage of individuals that had substitution of $\mathrm{C} \rightarrow \mathrm{A}$ at site 86-bp than the cultivated populations. At sites 10-bp, 37-bp, 42-bp and 235-bp, these bases of the Schisandra sphenanthera samples differed from that of $S$. chinensis. Two species showed higher levels of inter-specific divergence than intra-specific divergence within ITS2 sequences. However, 24 populations did not demonstrate much difference as inter-specific and intra-specific divergences were concerned. Both S. chinensis and S. sphenanthera showed monophyly at species level, yet the samples of different populations shown polyphyly at population level. ITS2 performed well when using BLAST1 method. ITS2 obtained 100\% identification success rates at the species level for S. chinensis, with no ambiguous identification at the genus level for ITS2 alone. The ITS2 region could be used to identify S. chinensis and S. sphenanthera in the "Chinese Pharmacopoeia". And it could also correctly distinguish 100\% of species and 100\% of genera from the 193 sequences of $S$. chinensis. Hence, the ITS2 is a powerful and efficient tool for species identification of S. chinensis.
\end{abstract}

Keywords: DNA barcode, ITS2, Schisandra chinensis, species, populations

\section{Introduction}

Schisandra chinensis (Turcz.) Baill. grows mainly in the northeast China and its mature fruits are famous traditional Chinese medicine recorded in "Chinese Pharmacopoeia". As a traditional medicinal herb, $S$. chinensis has been used as an astringent curing dry cough, asthma, night sweats, nocturnal seminal emissions and chronic diarrhea [1,2]. Modern medical research had proved that $S$. chinensis contains multiple active components used to protect liver [3-5], prevent senility [6-9], restrain oxidation $[10,11]$, improve human body immunity ability [12], regulate the central nervous system and so on $[13,14]$.

Commonly $S$. chinensis and its adulterants are frequently found in the market together. Schisandra sphenanthera Rehd. et Wils. is important traditional Chinese medicine recorded in "Chinese Pharmacopoeia", too, but its active components

* Corresponding author. Email: wangbing1616@163.com

Handling Editor: Przemysław Wojtaszek

This is an Open Access digital version of the article distributed under the terms of the Creative Commons Attribution 3.0 License (creativecommons.org/licenses/by/3.0/), which permits redistribution, commercial and non-commercial, provided that the article is properly cited. of the kinds and levels are clearly different from S. chinensis [15]. Conventional viewpoints are that the medicinal value of S. chinensis is better than S. sphenanthera. But the two medicines are often mistaken for each other [16]. The identification of the two species of Schisandra is difficult when based solely on morphological characteristics. Additionally, some limitations in traditional taxonomy prevent this technique from meeting the complicated demands of species recognition [17]. As such, a method for the simple and accurate authentication of Schisandra is indispensible.

As yet some works had been done to differentiation or identification of Schisandra species using RAPD, ISSR, $r b c L$ and ITS [18-20]. Recently, being part of ITS, ITS2 was relatively easy to be amplified using one pair of universal primers [21,22]. In addition, ITS2 had been found to provide taxonomic signature in systematic evolution $[23,24]$. The ITS2 region was also a promising potential molecular marker to be used for rapid taxonomic classification $[21,22]$. To our best knowledge, applying ITS2 region to identify plant materials from Schisandra with such a large sample size and geographic range had not been reported. And these studies were not found whether the ITS2 region could be used as a genomic marker to identify different Schisandra populations from different ecological environment and geographical distribution. In the current study, we utilized ITS2 as a DNA barcode to distinguish medicinal plants within the Schisandra genus and populations in order to ensure their safe, effective application in traditional using. 


\section{Material and methods}

\section{Plant materials}

In our study, 217 samples - 193 samples of S. chinensis and 24 samples of $S$. sphenanthera, which belonged to 24 populations from two species, were collected from 17 counties of nine provinces in China between June and August 2012 (Tab. 1). All subjects were identified by Professor Bing Wang, Liaoning University of Traditional Chinese Medicine, China. The voucher samples were deposited in the herbarium of Liaoning University of Traditional Chinese Medicine.

\section{DNA extraction, amplification and sequencing}

Genomic DNA was extracted from silica gel-dried leaves according to the protocol associated with the Plant Genomic DNA Kit (Tiangen Biotech Co., China). Polymerase chain reaction (PCR) amplification of the ITS2 region was carried out in the Peltier Thermal Cycler PTC0200 (BioRad Lab Inc., USA) using approximately $30 \mathrm{ng}$ of genomic DNA as a template in a $25-\mu \mathrm{l}$ reaction mixture $\left[1 \times \mathrm{PCR}\right.$ buffer without $\mathrm{MgCl}_{2}, 2.0 \mathrm{mM} \mathrm{MgCl}_{2}$, $0.2 \mathrm{mM}$ of each dNTP, $0.1 \mu \mathrm{M}$ of each primer (synthesized by Sangon Co., China)], and 1.0 U of Taq DNA Polymerase (Biocolor BioScience \& Technology Co., China). ITS2 primer and PCR amplification for the ITS2 region were conducted as described previously [25]. The PCR products were run on a $1.2 \%$ agarose gel in $0.5 \times$ TBE buffer and purified with the TIANGel Midi Purification Kit (Tiangen Biotech Co., China). The purified PCR products were sequenced on an ABI 3730XL sequencer (Applied Biosystems Inc.) using the amplification primers.

\section{Sequence alignment and data analysis}

Contig assembly and the generation of consensus sequences were performed using CodonCode Aligner ver. 3.0 (CodonCode Co., USA). The ITS2 sequences from GenBank were subjected to hidden Markov model (HMM) model analysis of HMMer annotation to remove the conserved 5.8S and 26S (or equivalent) rRNA sequences [26-28]. And we also used HMMer based annotation on well-curated fungal sequences to search for downloaded ITS2 sequences to remove the possible contaminated sequences of fungi. The sequences were then aligned using Clustal W and the genetic distances were computed using MEGA 5.0 according to the Kimura 2-parameter (K2P) model $[29,30]$. The average intra-specific distance, coalescent depth and Theta were calculated to evaluate the intra-specific variation using the K2P model [22,31]. The average inter-specific distance, the minimum inter-specific distance and Theta prime were used to represent inter-specific divergences [22,31,32]. The distributions of intra- vs. inter-specific variability were compared using DNA barcoding gaps [22,31,33]. Wilcoxon two-sample tests were performed as described previously $[22,33,34]$. Two methods of species identification, including NJ tree and BLAST1-based methods, were performed as described previously [35]. In the BLAST1 method, correct identification means that the best basic local alignment search tool (BLAST) hits of the query sequence is from the expected species; ambiguous identification means that the best BLAST hits for a query sequence is found to be those of several species including the expected species; incorrect identification means that the best BLAST hits of the query sequence is not from the expected species.

Tab. 1 Plant samples of Schisandra used in the present study.

\begin{tabular}{|c|c|c|c|c|c|}
\hline Species & Population codes & Collected place & Statement & Number & Accession number \\
\hline Schisandra chinensis & BG & Donggang, Liaoning, China & Cultivated & BG1-8 & AB558158.1 \\
\hline \multirow{21}{*}{ (Turcz.) Baill. } & BJ & Yanqing, Beijing, China & Wild & BJ1-2 & AB558158.1 \\
\hline & $\mathrm{CH}$ & Wangqing, Jilin, China & Cultivated & $\mathrm{CH} 1-10$ & AB558158.1 \\
\hline & FX & Fengcheng, Liaoning, China & Cultivated & FX1-4 & AB558158.1 \\
\hline & $\mathrm{KD}$ & Kuandian, Liaoning, China & Wild & KD1-10 & AB558158.1 \\
\hline & HG & Donggang, Liaoning, China & Cultivated & HG1-5 & AB558158.1 \\
\hline & $\mathrm{LH}$ & Fusong, Jilin, China & Wild & LH1-10 & AB558158.1 \\
\hline & LJ & Linjiang, Jilin, China & Wild & LJ1-10 & AB558158.1 \\
\hline & MJ & Mudanjiang, Heilongjiang, China & Wild & MJ1-10 & AB558158.1 \\
\hline & NM & Jiagedaqi, Neimenggu, China & Wild & NM1 & AB558158.1 \\
\hline & QC & Cixiangguan & Wild & QC1-10 & AB558158.1 \\
\hline & QS & Liaoning, China & Wild & QS1-10 & AB558158.1 \\
\hline & QX & Xianrentai & Wild & QX1-10 & AB558158.1 \\
\hline & $S$ & Tieli, Heilongjiang, China & Wild & S1-10 & AB558158.1 \\
\hline & SZ & Tieli, Heilongjiang, China & Cultivated & SZ1-10 & AB558158.1 \\
\hline & $\mathrm{TP}$ & Chicheng, Hebei, China & Wild & TP1-10 & AB558158.1 \\
\hline & WQ & Wangqing, Jilin, China & Wild & WQ1-10 & AB558158.1 \\
\hline & YS & Panshi, Jilin, China & Wild & YS1-10 & AB558158.1 \\
\hline & YT & Yantai, Shandong, China & Wild & YT1-15 & AB558158.1 \\
\hline & $\mathrm{ZE}$ & Fengcheng, Liaoning, China & Cultivated & ZE1-8 & AB558158.1 \\
\hline & $\mathrm{ZH}$ & Zhuanghe, Liaoning, China & Wild & ZH1-10 & AB558158.1 \\
\hline & $\mathrm{ZZ}$ & Fengcheng, Liaoning, China & Cultivated & ZZ1-10 & AB558158.1 \\
\hline Schisandra & LB & Lingbao, Henan, China & Wild & LB2-15 & AF263437.1 \\
\hline $\begin{array}{l}\text { sphenanthera Rehd. } \\
\text { et Wils. }\end{array}$ & PL & Pinglu, Shanxi, China & Wild & PL1-10 & AF263437.1 \\
\hline
\end{tabular}


Results

\section{Analysis of the sites mutation of ITS2 sequences}

Base mutation at site 86-bp was found in large quantities of samples in S. chinensis populations. And the wild populations had higher percentage of individuals that had substitution of $\mathrm{C} \rightarrow \mathrm{A}$ at site 86-bp than the cultivated populations, Qianshan in Liaoning province 63\%, WQ 60\% and TP 70\%. Also, base mutation at sites 58-bp and 227-bp were found in samples of LB13, PL1 and PL7 in S. sphenanthera populations, but the S. chinensis populations were not found (Tab. 1, Tab. 2). At sites 10-bp, 37-bp, 42-bp and 235-bp, these bases of all the $S$. sphenanthera samples differed from the samples of $S$. chinensis. So the samples of $S$. chinensis and S. sphenanthera could be distinguished accurately based on these base sites (Tab. 3).

Tab. 2 The sites mutation in the samples of $S$. chinensis and $S$. sphenanthera.

\begin{tabular}{|c|c|c|c|c|c|}
\hline Site & 58-bp & 86-bp & 183-bp & 227-bp & 229-bp \\
\hline BG & $\mathrm{C}$ & $\mathrm{C}$ & G & $\mathrm{C}$ & G \\
\hline $\mathrm{CH} 1,3$ & & A & & & \\
\hline $\mathrm{FX} 2,3$ & & A & & & \\
\hline HG1,4 & & A & & & \\
\hline LH2,5 & & A & & & \\
\hline LJ7,8 & & A & & & \\
\hline $\mathrm{QC} 1,4,5,6,7,8,9$ & & A & & & \\
\hline QS2,3,5,9 & & A & & & \\
\hline QX1,2,4,5,6,8,9,10 & & A & & & \\
\hline$S Z 6,7,8,10$ & & A & & & \\
\hline $\mathrm{TP} 1,2,3,4,7,8,10$ & & A & & & \\
\hline WQ4,5,6,7,8,9, & & A & & & \\
\hline YS2, 5,7,9 & & A & & & \\
\hline ZE3 & & A & & & \\
\hline ZH6,9 & & A & & & \\
\hline ZZ6 & & A & & & \\
\hline KD2 & & & $\mathrm{T}$ & & \\
\hline $\mathrm{ZZ2}$ & & & A & & $\mathrm{T}$ \\
\hline LB13 & $\mathrm{T}$ & & & & \\
\hline PL1,7 & $\mathrm{T}$ & & & $\mathrm{T}$ & \\
\hline
\end{tabular}

The ITS2 sequences of the samples of BG population stands for consensus ITS2 sequences of $S$. chinensis and S. sphenanthera.

Tab. 3 The different sites between the samples of S. chinensis and S. sphenanthera.

\begin{tabular}{|c|c|c|c|c|}
\hline Sites & 10-bp & 37-bp & 42-bp & 235-bp \\
\hline BG & A & $\mathrm{T}$ & G & $\mathrm{T}$ \\
\hline LB & $\mathrm{T}$ & C & $\mathrm{T}$ & A \\
\hline PL & $\mathrm{T}$ & C & $\mathrm{T}$ & A \\
\hline
\end{tabular}

The ITS2 sequences of the samples in BG population stands for consensus ITS2 sequences of $S$. chinensis.

\section{Measurement of DNA divergence for ITS2}

The lengths of the ITS2 sequences used for the analyses were $231 \mathrm{bp}$. We used six metrics to characterize inter- vs. intra-specific variations in ITS2 sequences [22,31,32]. As shown in Tab. 4, species showed significant levels of inter-specific divergence within ITS2 sequences. Relatively lower levels of intra-specific divergence were found with calculations for the three metrics. Therefore, the ITS2 region of the Schisandra species, with lower levels of genetic divergence within species than between species, again showed that might be used as a genomic marker for the identification of the two species.

To evaluate the reliability that the ITS2 region could be used as a genomic marker for the identification of the different populations in Schisandra, we also characterized the intra- and inter-specific variations in ITS2 sequences. At population level, we used the same six metrics to characterize inter- vs. intraspecific variations. As shown in Tab. 4, the populations showed levels of inter-specific divergence within ITS2 sequences were fairly near the levels of intra-specific divergence that were found with calculations for the three metrics except Coalescent depth. Wilcoxon two-sample tests also showed no significant difference between the mean of the inter-specific divergences and the intra-specific variations in populations (Tab. 5). Based on the results of this experiment, the ITS2 region of the Schisandra did not possess apparent intra- and inter-specific variation gaps of these populations with the two species. The ITS2, with the same genetic divergence within populations and among populations, should not be used as a genomic marker for identifying the populations of S. chinensis and S. sphenanthera.

Tab. 4 Analyses of inter-specific divergence between congeneric species, populations and intra-specific variations in ITS2 sequences in Schisandra.

\begin{tabular}{lcl}
\hline \multicolumn{1}{c}{ Measurement } & Species & Populations \\
\hline All inter-specific distance & $0.0236 \pm 0.0381$ & $0.0070 \pm 0.0157$ \\
Theta prime & $0.0236 \pm 0.0381$ & $0.0061 \pm 0.0013$ \\
The minimum inter-specific distance & $0.0236 \pm 0.0381$ & $0.0037 \pm 0.0111$ \\
All intra-specific distance & $0.0078 \pm 0.0550$ & $0.0070 \pm 0.0520$ \\
Theta & $0.0064 \pm 0.0019$ & $0.0067 \pm 0.0228$ \\
Coalescent depth & $0.0217 \pm 0.1184$ & $0.0294 \pm 0.1147$ \\
& & \\
& & \\
& &
\end{tabular}

\section{Assessment of the intra- vs. inter-specific differences of ITS2 sequences}

To perform a preliminary examination of inter- and intraspecific variation, we investigated the distribution of genetic distance in classes of 0.006 distance units. Only a slight overlap in inter/intra-specific variation of the ITS2 was found in our study (Fig. 1a). The inter-specific distance equaled to zero for $0 \%$ of the samples. Also, most of the Schisandra species in some studies were found to have a unique sequence in the ITS2 [36,37]. This will provide a useful way to authenticate different ITS2 species. To perform a preliminary examination of inter- and intra-specific variation among the populations of the Schisandra, then we studied the distribution of genetic distance too. And notable overlap in inter/intra-specific variation of ITS2 was found (Fig. 1b). The inter- and intra-specific distance less than 0.006 reached for $97.45 \%$ and $95.25 \%$ of the samples with these populations. 
Tab. 5 Wilcoxon two-sample tests for distribution of intra- vs. interspecific divergences.

\begin{tabular}{lcccc} 
Data sources & $\begin{array}{c}\text { No. of inter- } \\
\text { specific } \\
\text { distances }\end{array}$ & $\begin{array}{c}\text { No. of intra- } \\
\text { specific } \\
\text { distances }\end{array}$ & Wilcoxon W & P value \\
\hline species & 1 & 2 & $\#$ & $\#$ \\
populations & 232 & 24 & 2498.0 & 0.185 \\
\hline
\end{tabular}

“\#”stands for species were not analyzed, because the No. of inter- vs. intra-specific distances were few.

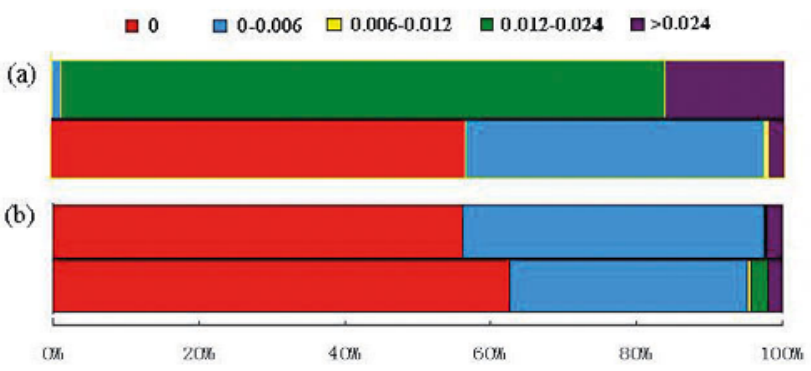

Fig. 1 Relative distribution of inter-specific divergences between congeneric species/population and intra-specific variations for ITS2 sequences. The colored bars in each box represent inter-specific (above) and intra-specific (below) genetic distances. a Species. b Population.

\section{$\mathrm{NJ}$ tree and BLAST1-based methods were used to identify the samples}

Two dendrograms were constructed on account of neighbor joining (NJ; Fig. 2). The same species were classed together obviously. The species of $S$. chinensis and S. sphenanthera all showed monophyly, and the two species could be differentiated obviously (Fig. 2a). The samples of different populations showed polyphyly at population level. And the samples of different populations were classed together, S. sphenanthera populations, for example (Fig. 2b). So the samples of different populations and geographical origins could not be differentiated by NJ tree-based method.

ITS2 performed well when using BLAST1 method. ITS2 obtained $100 \%$ identification success rate at the species level for S. chinensis, with no ambiguous identification at the genus level for ITS2 alone. S. sphenanthera, the success rate of ITS2 was $0 \%$ at the species level, but reached up to $100 \%$ at the genus level.

\section{Discussion}

A rapid and accurate method to authenticate species from the family Schisandraceae is very important to ensure the safe, effective usage of drugs made from the two medicinal herbs. To our knowledge, this was the first time that the ITS2 region was used to identify plant materials from Schisandra with such a large sample size and geographic range. In the study, ITS2 was found to be a sufficiently variable DNA region between $S$. chinensis and S. sphenanthera species as determination by genetic divergences, and ITS2 also demonstrated a high capability of successful discrimination. ITS2 can be a powerful marker for taxonomy studies, identifying species and solving taxonomic problems.

With the wide distribution and quite variable leaf shape, S. sphenanthera was mistaken for S. chinensis more often [16]. And it was hard to identify the two commercial products too. ITS2 sequences of $S$. chinensis were different from that of S. sphenanthera samples by 4 bases. Therefore, the molecular results supported the view of the traditional taxonomists and could distinguish the two species accurately. When identifying S. chinensis by NJ tree-based method, the 193 samples of $S$. chinensis were clustered as a clade. And all samples of S. chinensis were successfully identified using BLAST1-based method. In the study, ITS2 obtained 0\% identification success rate at the species level for $S$. sphenanthera, with no ambiguous identification at the genus level for ITS2 alone by BLAST1-based method. But the two species of S. chinensis and S. sphenanthera could be distinguished by NJ tree and BLAST1-based methods.

When identifying S. sphenanthera by BLAST1-based method, the best BLAST hit of the query sequence of the samples with $S$. sphenanthera contain several species - Schisandra viridis A. C. Smith, Schisandra glaucescens Diels in Bot. and Schisandra rubriflora (Franch.) Rehd. et Wils. S. sphenanthera is distributed mainly over Shanxi, Henan, Hubei, Hunan, Sichuan, Anhui, Zhejiang, Jiangxi and Guizhou provinces, China. A point worth emphasizing was that these samples of S. sphenanthera were collected by members of task group in Lingbao city Henan province and Pinglu city Shanxi province, China, and the species and sources were accurate, definite. The best BLAST hit of the query sequence of the samples with $S$. sphenanthera were not our expectation for a few reasons. First, we could easily found these species overlaped in geographical distribution [16]. So these species could produce gene flows in the cross areas each other, theoretically. And the divergence, in a way, was reduced; Second, ITS2 cannot solve all the species determination problems. For example, in Caragana, Caragana tibetica and Caragana ordosica were found to have identical ITS2 sequences [38-42], but they were already reported to be two different species based
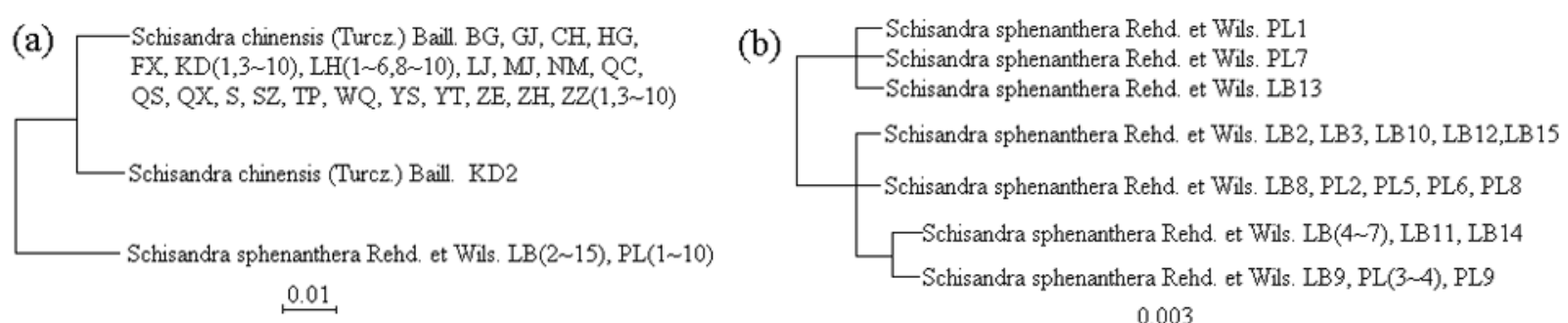

0.003

Fig. 2 The NJ tree method on account of ITS2 sequences. a The two species of Schisandra chinensis and S. sphenanthera. b The populations with $S$. sphenanthera. 
on their ITS sequences [40]. Thus, other DNA marker(s) might be valuable when investigating certaingenus and broad plant taxa such that complete species identification could be achieved in Schisandra, psbA-trnH [33,43-45], for instance.

The ITS2 region of the Schisandra species could be used as a genomic marker for the identification of the two species with $S$. chinensis and S. sphenanthera, but could not be used as a genomic marker to identify different populations or origins of the two species. The present study showed the different populations of the two species were found to have identical ITS2 sequences, respectively. It could only make a primary judgment of the species' origin with the wild or cultivated samples based on test results.

\section{Conclusion}

In this study, ITS2 was examined for its usefulness in identifying medicinal species of Schisandra. Our findings showed that the ITS2 region could be used to identify S. chinensis and S. sphenanthera in the "Chinese Pharmacopoeia". And could also correctly distinguish $100 \%$ of species and $100 \%$ of genera from the 193 sequences of $S$. chinensis. Hence, ITS2 is a powerful and efficient tool for species identification of medicinal plants and even for a broad series of Schisandra plant taxa.

\section{Acknowledgments}

This work was supported by the special, key projects of the basic work with the Nation and Technology - the investigation of the resources with endangered and most large medicinal plants (No. 2007FY1106002010) and the items with Liaoning Department of Education, China (No. LT2010067).

\section{Authors' contributions}

The following declarations about authors' contributions to the research have been made: research designing: BW; conducting experiments: XL, YZ, HY, LX, JZ, BX; analyzed the data and wrote the paper: XL, RH.

\section{References}

1. Saunders RMK. Monograph of Kadsura (Schisandraceae). Syst Bot Mon. 1998;54:24-106. http://dx.doi.org/10.2307/25096646

2. Lin Q, Duan LD, Yao BF. Notes on three species of the genus Kadsura Juss. (Schisandraceae). Acta Phys Sin. 2005;43(6):567-570. http://dx.doi. org/10.1360/aps030102

3. Teraoka R, Shimada T, Aburada M. The molecular mechanisms of the hepatoprotective effect of gomisin A against oxidative stress and inflammatory response in rats with carbon tetrachloride-induced acute liver injury. Biol Pharm Bull. 2012;35(2):171-177. http://dx.doi.org/10.1248/bpb.35.171

4. Ip SP, Poon MKT, Wu SS, Che CT, Ng KH, Kong YC, et al. Effect of schisandrin B on hepatic glutathione antioxidant system in mice: protection against carbon tetrachloride toxicity. Planta Med. 2007;61(05):398-401. http://dx.doi.org/10.1055/s-2006-958123

5. Ip SP, Ko KM. The crucial antioxidant action of schisandrin B in protecting against carbon tetrachloride hepatotoxicity in mice: a comparative study with butylated hydroxytoluene. Biochem Pharmacol. 1996;52(11):16871693. http://dx.doi.org/10.1016/S0006-2952(96)00517-5
6. Nishiyama N, Chu PJ, Saito H. An herbal prescription, S-113m, consisting of biota, ginseng and schizandra, improves learning performance in senescence accelerated mouse. Biol Pharm Bull. 1996;19(3):388-393. http://dx.doi.org/10.1248/bpb.19.388

7. Kang SY, Lee KY, Koo KA, Yoon JS, Lim SW, Kim YC, et al. ESP-102, a standardized combined extract of Angelica gigas, Saururus chinensis and Schizandra chinensis, significantly improved scopolamine-induced memory impairment in mice. Life Sci. 2005;76(15):1691-1705. http:// dx.doi.org/10.1016/j.lfs.2004.07.029

8. Hsieh MT, Wu CR, Wang WH, Lin LW. The ameliorating effect of the water layer of fructusschisandrae on cycloheximide-induced amnesia in rats: interaction with drugs acting at neurotransmitter receptors. Pharmacol Res. 2001;43(1):17-22. http://dx.doi.org/10.1006/phrs.2000.0756

9. Hsieh MT, Tsai ML, Peng WH, Wu CR. Effects of Fructus schizandrae on cycloheximide-induced amnesia in rats. Phytother Res. 1999;13(3):256-257. http://dx.doi.org/10.1002/ (SICI)1099-1573(199905)13:3<256::AID-PTR435>3.0.CO;2-H

10. Sheng Y, Liu Y, Huang XD, Yuan GX, Guan M. Purification, chemical characterization and in vitro antioxidant activities of alkali-extracted polysaccharide fractions isolated from the fruits of Schisandra chinensis. J Med Plants Res. 2011;5(24):5881-5888.

11. Jung GT, Ju IO, Choi JS, Hong JS. The antioxidative, antimicrobial and nitrite scavenging effects of Schisandra chinensis RUPRECHT (Omija) seed. Korean J Food Sci Technol. 2000;32:928-935.

12. Mizoguchi Y, Shin T, Kobayashi K, Morisawa S. Effect of gomisin A in an immunologically-induced acute hepatic failure model. Planta Med. 1991;57(1):11-14. http://dx.doi.org/10.1055/s-2006-960006

13. Fu M, Sun ZH, Zong M, He XP, Zuo HC, Xie ZP. Deoxyschisandrin modulates synchronized $\mathrm{Ca}^{2+}$ oscillations and spontaneous synaptic transmission of cultured hippocampal neurons. Acta Pharmacol Sin. 2008;29(8):891-898. http://dx.doi.org/10.1111/j.1745-7254.2008.00821.x

14. Kim SR, Lee MK, Koo KA, Kim SH, Sung SH, Lee NG, et al. Dibenzocyclooctadiene lignans from Schisandra chinensis protect primary cultures of rat cortical cells from glutamate-induced toxicity. J Neurosci Res. 2004;76(3):397-405. http://dx.doi.org/10.1002/jnr.20089

15. Hu YJ, Chen JZ, Ye L. The study evolvement of chemic components and differentiation methods between Schisandra chinensis and Schisandra sphenanthera. Res Pr Chin Med. 2008;22(4):59-62.

16. Law YW, editor. Menispermaceae \& Magnoliaceae. Beijing: Chinese Academy of Sciences; 1996. (vol 30).

17. Maddison DR, Schulz KS, Maddison WP. The tree of life web project. Zootaxa. 2007;1668:19-40.

18. Wang PX, Li JY, Zhou L. Identification of S. chinensis (Turcz.) Baill. (Beiwuwei) and S. sphenanthera Rehd. et Wils. (Nanwuwei) by random amplified polymorphic DNA. Tradit Chin Drug Res Clin Pharmacol. 2002;13(2):98-99.

19. Sun $Y$, Wen X, Huang H. Population genetic differentiation of Schisandra chinensis and Schisandra sphenanthera as revealed by ISSR analysis. Biochem Syst Ecol. 2010;38(3):257-263. http://dx.doi.org/10.1016/j. bse.2010.01.005

20. Kim JS, Jang HW, Kim JS, Kim HJ, Kim JH. Molecular identification of Schisandra chinensis and its allied species using multiplex PCR based on SNPs. Genes Genom. 2012;34(3):283-290. http://dx.doi.org/10.1007/ s13258-011-0201-3

21. Chiou SJ, Yen JH, Fang CL, Chen HL, Lin TY. Authentication of medicinal herbs using PCR-amplified ITS2 with specific primers. Planta Med. 2007;73(13):1421-1426. http://dx.doi.org/10.1055/s-2007-990227

22. Chen S, Yao H, Han J, Liu C, Song J, Shi L, et al. Validation of the ITS2 region as a novel DNA barcode for identifying medicinal plant species. PLoS ONE. 2010;5(1):e8613. http://dx.doi.org/10.1371/journal.pone.0008613

23. Coleman AW. ITS2 is a double-edged tool for eukaryote evolutionary comparisons. Trends Genet. 2003;19(7):370-375. http://dx.doi.org/10.1016/ S0168-9525(03)00118-5 
24. Coleman AW. Pan-eukaryote ITS2 homologies revealed by RNA secondary structure. Nucleic Acids Res. 2007;35(10):3322-3329. http://dx.doi. org/10.1093/nar/gkm233

25. Schultz J, Maisel S, Gerlach D, Müller T, Wolf M. A common core of secondary structure of the internal transcribed spacer 2 (ITS2) throughout the Eukaryota. RNA. 2005;11(4):361-364. http://dx.doi.org/10.1261/ rna.7204505

26. Eddy SR. Profile hidden Markov models. Bioinformatics. 1998;14(9):755763. http://dx.doi.org/10.1093/bioinformatics/14.9.755

27. Eddy SR. HMMER: profile hidden Markov models for biological sequence analysis. Wash Univ Med Alumni Q; 2000.

28. Keller A, Schleicher T, Schultz J, Müller T, Dandekar T, Wolf M. 5.8S-28S rRNA interaction and HMM-based ITS2 annotation. Gene. 2009;430(12):50-57. http://dx.doi.org/10.1016/j.gene.2008.10.012

29. Thompson JD, Higgins DG, Gibson TJ. CLUSTAL W: improving the sensitivity of progressive multiple sequence alignment through sequence weighting, position-specific gap penalties and weight matrix choice. Nucl Acids Res. 1994;22(22):4673-4680. http://dx.doi.org/10.1093/ nar/22.22.4673

30. Tamura K, Dudley J, Nei M, Kumar S. MEGA4: molecular evolutionary genetics analysis (MEGA) software version 4.0. Mol Biol Evol. 2007;24(8):1596-1599. http://dx.doi.org/10.1093/molbev/msm092

31. Meyer CP, Paulay G. DNA barcoding: error rates based on comprehensive sampling. PLoS Biol. 2005;3(12):e422. http://dx.doi.org/10.1371/journal. pbio.0030422

32. Meier R, Zhang G, Ali F. The use of mean instead of smallest interspecific distances exaggerates the size of the "barcoding gap" and leads to misidentification. Syst Biol. 2008;57(5):809-813. http://dx.doi. org/10.1080/10635150802406343

33. Lahaye R, van der Bank M, Bogarin D, Warner J, Pupulin F, Gigot G, et al. DNA barcoding the floras of biodiversity hotspots. Proc Natl Acad Sci USA. 2008;105(8):2923-2928. http://dx.doi.org/10.1073/pnas.0709936105

34. Kress WJ, Erickson DL. A two-locus global DNA barcode for land plants: the coding $r b c L$ gene complements the non-coding $t r n H$-psbA spacer region. PLoS ONE. 2007;2(6):e508. http://dx.doi.org/10.1371/journal. pone. 0000508
35. Ross HA, Murugan S, Li WLS. Testing the reliability of genetic methods of species identification via simulation. Syst Biol. 2008;57(2):216-230. http://dx.doi.org/10.1080/10635150802032990

36. Li DZ, Gao LM, Li HT, Wang H, Ge XJ, Liu JQ, et al. Comparative analysis of a large dataset indicates that internal transcribed spacer (ITS) should be incorporated into the core barcode for seed plants. Proc Natl Acad Sci USA. 2011;108(49):19641-19646. http://dx.doi.org/10.1073/pnas.1104551108

37. Liu Z, Wang XQ, Chen ZD, Lin Q, Lu AM. The phylogeny of Schisandraceae inferred from sequence analysis of the nrDNA ITS region. Acta Bot Sin. 2000;42(7):758-761.

38. Ma CC, Gao YB, Guo HY, Wang JL. Interspecific transition among Caragana microphylla, C. davazamcii and C. korshinskii along geographic gradient. II. Characteristics of photosynthesis and water metabolism. Acta Bot Sin. 2003;45(10):1228-1237.

39. Hou X, Liu JE, Zhao YZ, Zhao LQ. Interspecific relationships of Caragana microphylla, C. davazamcii and C. korshinskii (Leguminosae) based on ITS and trnL-F data sets. Acta Phytotaxon Sin. 2006;44(2):126-134. http:// dx.doi.org/10.1360/aps040077

40. Hou X, Liu JE, Zhao YZ. Molecular phylogeny of Caragana (Fabaceae) in China. Acta Phytotaxon Sin. 2008;46:600-607. http://dx.doi.org/10.3724/ SP.J.1002.2008.07071

41. Wojciechowski MF, Sanderson MJ, Baldwin BG, Donoghue MJ. Monophyly of aneuploid Astragalus (Fabaceae): evidence from nuclear ribosomal DNA internal transcribed spacer sequences. Am J Bot. 1993;80(6):711-722. http://dx.doi.org/10.2307/2445441

42. Zhang ML. Ancestral area analysis of the genus Caragana (Leguminosae). Acta Bot Sin. 2004;46(3):253-258.

43. Kress WJ, Wurdack KJ, Zimmer EA, Weigt LA, Janzen DH. Use of DNA barcodes to identify flowering plants. Proc Natl Acad Sci USA. 2005;102(23):8369-8374. http://dx.doi.org/10.1073/pnas.0503123102

44. Chase MW, Cowan RS, Hollingsworth PM, van den Berg C, Madriñán $\mathrm{S}$, Petersen $\mathrm{G}$, et al. A proposal for a standardised protocol to barcode all land plants. Taxon. 2007;56(2):295-299.

45. Newmaster SG, Fazekas AJ, Steeves RAD, Janovec J. Testing candidate plant barcode regions in the Myristicaceae. Mol Ecol Resour. 2008;8(3):480-490. http://dx.doi.org/10.1111/j.1471-8286.2007.02002.x 